\title{
Intelligent Music Abstraction Tool for improvising the Quality of Music Composition
}

\author{
Sudipta Chakrabarty ${ }^{1}$, Md Ruhul Islam ${ }^{2}$, Hiren kumar Deva Sarma ${ }^{3}$ \\ ${ }^{1}$ Department of Master of Computer Application, Techno Main Salt Lake, India, chakrabarty.sudipta@ gmail.com \\ ${ }^{2}$ Department of Computer Science \& Engineering, SMIT, Sikkim, India, ruhulislam786@ gmail.com \\ ${ }^{3}$ Department of Information Technology, SMIT, Sikkim, hirenkdsarma@ gmail.com
}

\begin{abstract}
All type of music pieces consist of two vital elements - rhythm structure and melody involvement. Another most important thing for automatic music improvisation is cadences that give symphonic shape to a music melody. Intelligent quality music composition is the relation of different musical elements and aggregation entity of these musical element objects. This paper introduces a new method for composing music using abstraction mechanism concept of software engineering. This paper focuses on the two main objects of music - Vocal and Instrumental and these two objects are tightly coupled and create the aggregation entity of Music Composition. The primary objective of this work is to explore the efficiency of Abstraction mechanism Generalization, Specialization, and Aggregation to search for an optimum combination between vocal and different instrumentals with their different tempo to improve the quality of music and versatile intelligent music composition.
\end{abstract}

Key words: Music Composition, Abstraction, Aggregation, Indian Classical Music, Music Information Retrieval, Unified Modeling Language

\section{INTRODUCTION}

The Classical Indian Music (ICM) is not as well-liked as the western music modeling [4], [7]. ICM is fully based on the arrangement of different note structures and note structures are unique for every music origin or raga. Hence, the recognition of notes used in Indian Classical vocal tunes and instruments is in fact a harsh task and the musical gestures can never be chased the linear expressions barely. Even though, the modern skills are by now given the soft and spiky thought in the field of musical pattern appreciation with some of the unusual characteristics like Indian Classical Raga, Thhats or raga origin, sequences of notes and their several characteristics [13], [15]. The recent advances of technology also furnish the musical characteristics by using less complicated approach such as Object-Oriented Analysis and modeling perspective [8], [9] [10], [11], [12]. The Artificial
Intelligence and Soft Computing approaches are also exploited for exact computations of the vocal features and their corresponding musical instruments such as musical rhythms utilized to act as ornaments on the vocal performances owing to be pointed out that the complexity and simplicity of systems to achieve practicability, robustness, and less effort of solutions [20], [24].

Object-Oriented Programming technology is a modus operandi where a set of substances can be categorized into several classes of numerous distinctive properties and appearances. All the objects of the analogous features are used to place in the identical class and the set of objects of dissimilar properties are categorized into other types of classes which are not similar depending on their behavior [18], [19]. A subclass depicts the configuration and behavior of objects that are entirely compatible with their super-class if the sub-classes have the similar types of arguments area as the super-class and, for all procedures of the super-class, related arguments are capitulated corresponding outcomes. Absolute compatibility authorizes instances of the subclasses to be freely influenced as instances of the super-class with no terror of unacceptable behavior [27], [30]. The concepts of classifications of the data, features or objects into several classes and subclasses lead to diminish the complexities of recognizing the features of the large systems. One fundamental abstraction mechanisms is Aggregation which is combining the features of information to create a new high level feature. In this work, Vocal and Instrument is two low level features are combining to create a high level feature Music Composition. The paper introduces the software Intelligent Music Abstraction Tool (IMAT) which provides music composition and performance improvisation system based on the concept of Software Engineering "Aggregation".

\section{RELATED WORK}

In Computer Computer-based Music Information Retrieval (MIR), music features have made of two basic classes - the vocal and instruments. Paper [1] explained that the relevance of rhythm structures and musical forms of a raga of ICM. In 
the paper [2] introduce a real-time system which listen of Tabla playing performances and identify the beat strokes. Another two papers used to stores the music information using symbolic score that is used for automatic transcription in solo performance in music [2], [3]. Two approaches that have been explained to the several researchers about the rhythm cycle complexities [4], [5]. In the same rhythm cycle perspective, the authors use the note structures of Tabla through automatic procedure [6]. Paper [7] represents about the mechanism of feature selection and extraction from different kinds of musical instruments [7]. Two more papers [10], [11], [12] deal with the implementation of Musical pattern recognition by mathematical expressions. The paper [13] has explained the notes structures with ten North Indian raga origin in ICM which is imposed to be constructed the songs. Another very important paper deals with the study of vocal performance and pitch analysis in Hindustani Classical Music (HCM) [14]. Some researchers were presenting the concept of Hindustani Music which automatically transforms into the acoustic signal from the song [15]. The authors of another paper introduced the concept of Statistical Pattern Recognition of musical signals using Artificial Neural Networks (ANN) [16]. Another paper introduced the concept of Computational Ethnomusicology [17]. Another interesting contribution represented as Unified Modeling Language (UML) diagrams are the way of implementations of implementation of musical rhythmic cycles is proved as the basis for the percussion-based instruments [8], [9].

In an effort by the authors have been introduced a Quality Music model used for ICM using the Roulette-Wheel Selection mechanism of Genetic Algorithm [20]. Three papers [21], [22] describe the usefulness of Genetic Algorithm in the field of music composition. These papers can also be applicable as an important tool in Musicology that can create versatile music composition as well as the versatile rhythm structure generations using the different operators of Genetic Algorithm. Another paper introduces a new concept that generates of realistic drum-set rhythms automatically using Genetic Algorithm [23]. Again introduces a new concept of variable length $n$-gram model in the paper that incorporates multiple views of Tabla bol sequences [24]. Some researchers introduce an automatic recognition system for recognition of the music patterns by using Recursive Median Filter [25]. Further one paper introduces the concept of Normalized Compression Distance method that is applicable for automatic music composition through the use of Genetic Algorithm [26]. Numerous contributions have been quite helpful to this work to provide sufficient information about data abstraction, generalization and specialization, inheritance, polymorphism and so on of the Object-Oriented methodologies and techniques [27], [28], [29], [30]. Rests are illustrated the performances to be replicated the Unified Modeling Language for the Object Technologies [31], [32], [33], [34], [35].
Petri nets is a modeling tool that is used as music pattern recognition and music pattern analysis for Indian Classical Music where object oriented methodology is the basis for musical pattern recognition and pattern analysis. The papers [36], [37] establish that the same concept in the field of Computational Musicology. The paper introduces a mechanism is that efficiently chooses the most fitted parent rhythms of a set of rhythm chromosomes for creating offspring rhythm using Genetic Algorithm Optimization technique in the context awareness pervasive music rhythm learning education Pervasive Education for Computational Musicology [38]. The paper [39] also identifies the raga name of a song music by mapping the with Raga Knowledgebase automatically. Another paper [40] has been introduced the concept of hummed query and user query depends on feedback of user that improve retrieval performance through Genetic Algorithm.

An approach that finds the matching similarity percentage between songs by their pitch values through Correlation of Coefficient [41]. The paper [42] represents a method to generate song list for listening, age factor of users for performing online. Again one paper represents the matching similarity between songs by their pitch values through Coefficient of variance [43]. Another paper proposes the time slot song music of a particular by using Neural Network [44] and the other work is based on Music Recommendation System that classifies different song clusters of different time of a day [45]. The paper [46] introduced an intelligent mechanism to identify the density of a given music rhythm and complexity of that music rhythm through software automatically. Paper [47] illustrates the different music research areas and their applications in the field of Computational Musicology. Another paper represents that a song of a particular raga can be represented through Unified Modelling Language [48]. Automatic Raga Recognition method identifies the notes of a song and then mapping with Raga database [49]. One interesting paper represents a survey work on low level, mid level, and track level feature extraction from music signal [50]. Another paper establishes the truth that Software Testing is the most important and essential phase of developing Software [51].

\section{FEATURE DETERMINATION AND TERMINOLOGIES}

In order to erect the piece of music, numerous fundamental musical features should be scrutinized. In Indian Classical Music (ICM) perspective, the vocal fundamental features like classical ragas and their corresponding classical Thhats are to be considered. In the Indian Classical rhythm domain, the actual set of notes or beats and its several sorts of variations of the corresponding rhythmic cycles imposed on the specific vocal performance. The primary attributes and features used in specifically in ICM which are to denote a specific music 
fundamentally are illustrated in the Table 1 . In this innovative contribution perspective, the musical abstraction analyzed the overview of this entire accomplishment of the fundamental musical features and their corresponding attributes.

Table 1: Different Musical Elements and their meanings

\begin{tabular}{|c|c|}
\hline $\begin{array}{l}\text { Musical } \\
\text { Elements }\end{array}$ & Meanings \\
\hline Beat & Beat is a fragment of note. \\
\hline Note & Notes can be a beat or combinations of beats. \\
\hline Meter or Matra & It is the length of a rhythmic structure. \\
\hline Tempo & Velocity of the vocal and instrumental music. \\
\hline Time Signature & $\begin{array}{l}\text { This is a notational convention to identify the number of } \\
\text { beat per measure. For } 4 / 4,3 / 4,2 / 4 \text {, and } 6 / 8 \text {. }\end{array}$ \\
\hline $\begin{array}{l}\text { Combining } \\
\text { Note Values }\end{array}$ & $\begin{array}{l}\text { Combinations of different notes in each measure. For } \\
\text { example } 4 / 4 .\end{array}$ \\
\hline Even Meters & $\begin{array}{l}\text { These are eventually divided by two and that is why also } \\
\text { called duple meter. }\end{array}$ \\
\hline Odd Meters & $\begin{array}{l}\text { These are eventually divided by three and that is why also } \\
\text { called triple meter. }\end{array}$ \\
\hline Raga & $\begin{array}{l}\text { Ragas are the basic element of music that is the unique } \\
\text { combinations of note structures. }\end{array}$ \\
\hline $\begin{array}{l}\text { Raga Origin or } \\
\text { Thhat }\end{array}$ & A thhat is the musical parent scale of different ragas. \\
\hline $\begin{array}{c}\text { Ascent \& } \\
\text { Descent }\end{array}$ & $\begin{array}{l}\text { The increasing pitch values of note sequences of a raga is } \\
\text { called and the decreasing pitch values of note sequences is } \\
\text { called Descent. }\end{array}$ \\
\hline $\begin{array}{l}\text { Aalap or } \\
\text { Rendition }\end{array}$ & $\begin{array}{l}\text { It is the melodic improvisation of classical music } \\
\text { performance for opening section. }\end{array}$ \\
\hline $\begin{array}{c}\text { Shrutis or } \\
\text { Linking Notes }\end{array}$ & $\begin{array}{l}\text { The smallest interval between different notes which are } \\
\text { interlinked between notes to other notes. }\end{array}$ \\
\hline Rhythm & It is a unit that accomplishes a set of the musical beats. \\
\hline Clap or Tali & It acts as physical notation. \\
\hline Wave or Khali & It also a physical notation which means null or off clap. \\
\hline Sam & The primary beat or note of a particular rhythmic cycle. \\
\hline Lay & The tempo in music is called the lay or laya. \\
\hline
\end{tabular}

\section{EXPERIMENTAL DETAILS}

Music composition using interactive systems is not very easy task; rather it is the evaluation metric by developer which is pleasant for listening. This paper introduces a qualitative approach that provides the versatile combinations of vocal and instruments opportunities among a set of combinations and improvising best quality music composition through abstraction mechanism. An abstraction combines a set of elements by omitting some details. Specially consider the three classical abstraction principles, generalization, specialization and aggregation. Aggregation is an abstraction principle that allows combining a set of parts to a whole. We are implementing these three concepts in our work. Unified Modeling Language (UML) is the part of the object-oriented software paradigm in a wide range of applications and it is one of the modeling languages of representing Abstraction mechanism. This paper represents a formal description of different musical elements through UML technologies and diagrams, mainly Class Diagram, Activity Diagram, Collaboration Diagram, and Sequence Diagram for visualized the specifications of our music composition in the context of Hindustani Music.

\subsection{Class Diagram}

Normally it describes how a system is structured to a certain extent than how it acts. The preliminary components of a class diagram are the classes and their relationships: Generalization, Aggregation, Association, and a variety of dependencies. Here in this Class diagram shows how the preliminary musical features are dependant and relationships with each other in Figure 1.

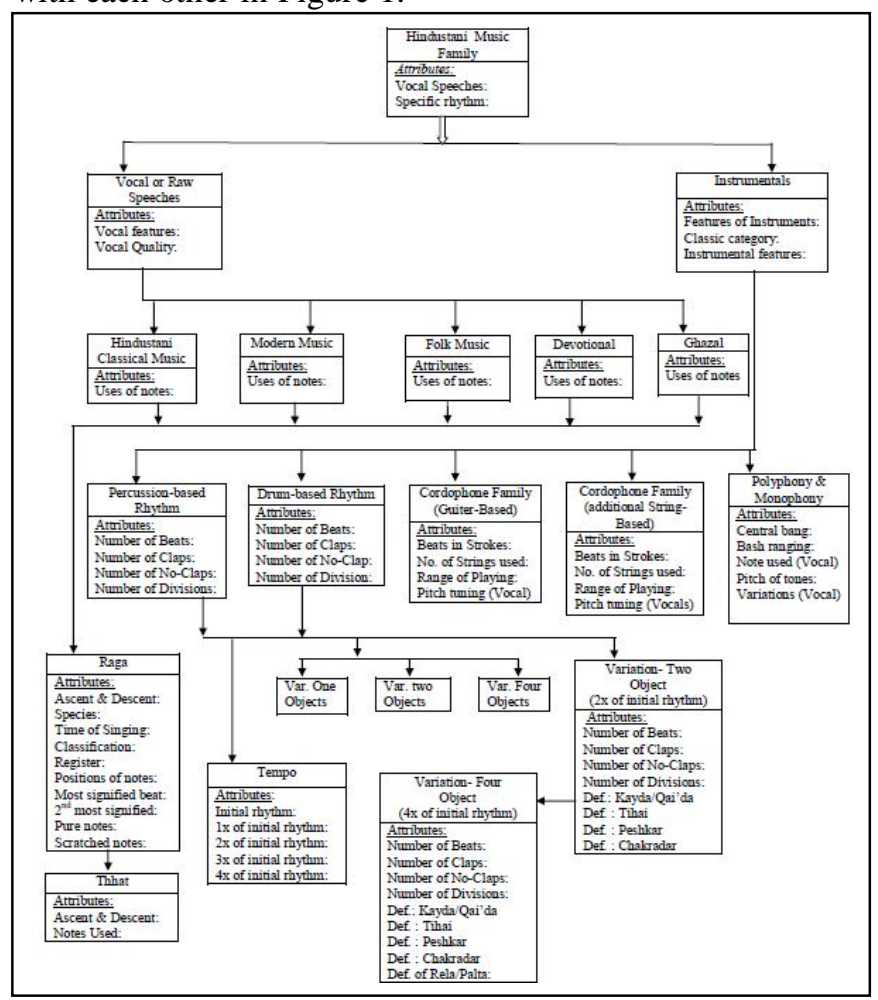

Figure 1: Class Diagram of IMAT

\subsection{Activity Diagram}

Generally activity diagram describes the state with an internal action and one or more outgoing alteration that repeatedly chase the termination of the internal activity. This diagram centers on representing the activities or mass of processing which may or may not communicate to the methods of classes. The Figure 2 shows the fraction participated by dissimilar components of any musical tool where initially the vocal raw 
data played by the vocalist. According to the vocal characteristics, the musical instruments are to be assigned by the users. After completion of all these activities the entire music can be generated and issued to the music library.

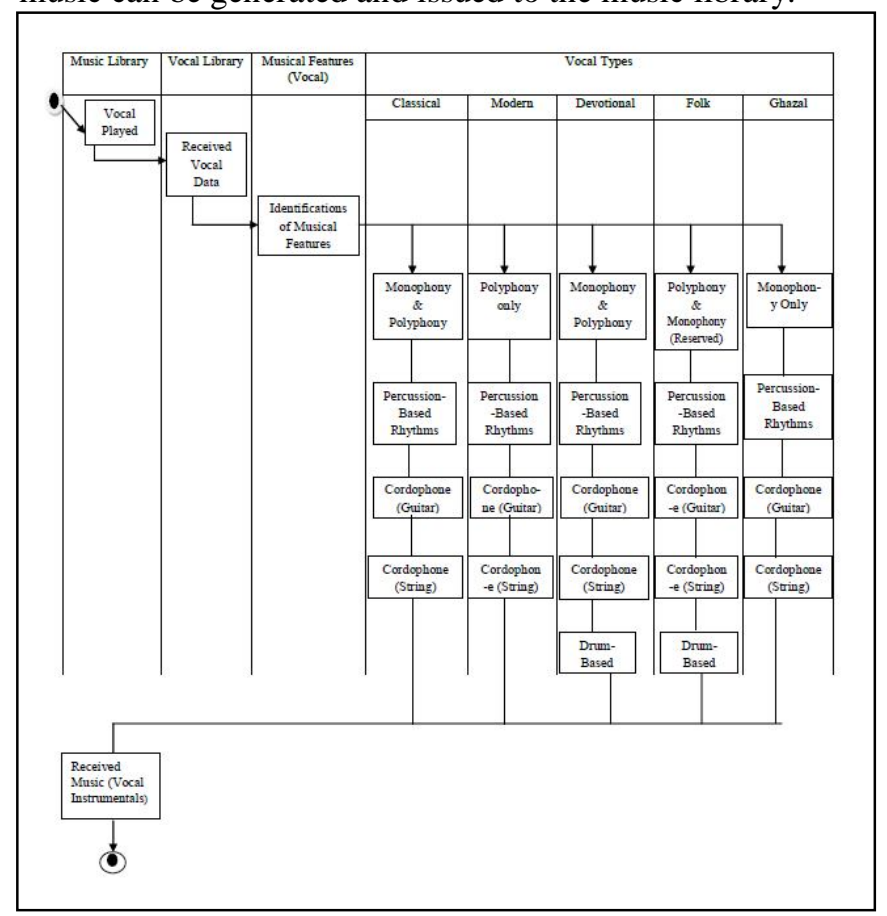

Figure 2: Activity Diagram of IMAT

\subsection{Collaboration Diagram}

A collaboration diagram displays both behavioral and structural characteristics unambiguously. The structural characteristic of a collaboration diagram consists of objects and the associations accessible between them. The figure modeled below would be to assist a designer to conclude which class is related or dependant with which class or classes in Figure 3.

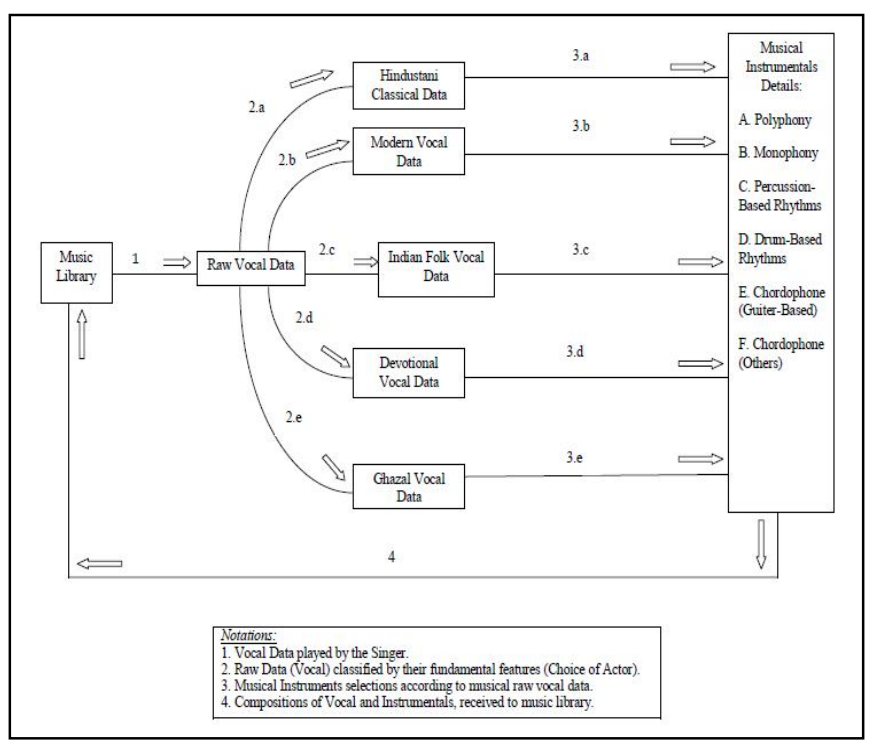

Figure 3: Collaboration Diagram of IMAT

\subsection{Sequence Diagram}

The sequence diagram demonstrates the interactions among objects as a two dimensional visual aid. The chart is to be read from peak to base. The objects contribute to the communication are to be displayed at the top of the graphic representation as boxes affixed to a vertical black stripe. The Sequence Diagram of our work is given in Figure 4.

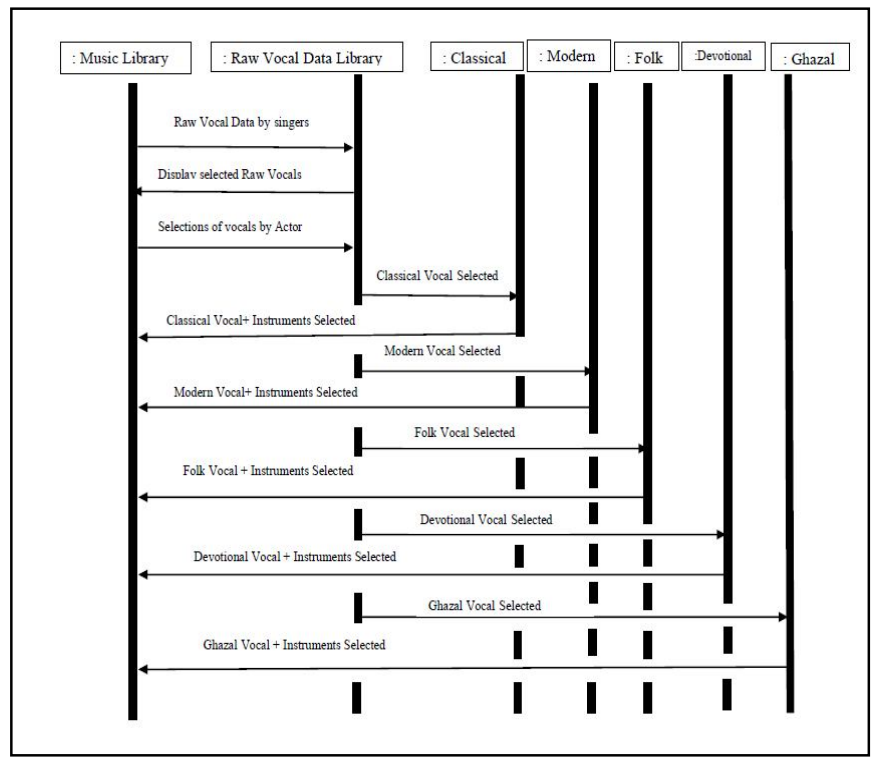

Figure 4: Sequence Diagram of IMAT

\section{EXPERIMENTAL DETAILS}

To establish the work, recorded a few vocal songs of different types using different singers and also recorded a set of musical instrumentals for every vocal performance separately. For that purpose, a lot of hardware devices are used, like Audiotechnica AT 2020, Cardioid Condenser Microphone, XLR Cable, and Sound Card: M-AUDIO FastTrackPro, etc. Java (JDK) 6.0 and JCreator IDE version 6 have been used for implementing the user interface design to establishing the working principles.

All the vocal files stored in a separate directory using the naming conventions 1.mp3, 2.mp3, 3.wav, etc. after recording of all the required data. IMAT provides user friendly or simple platform to user as it supports both .mp3 and .wav extension. We have also made a separate directory for different instrumentals using another naming convention, like 1Drum based.mp3, 1Monophony based.mp3, 1Percusion based.mp3, 1Polyphony based.mp3, 1String based.mp3, 1Woodwind based.mp3. For .wav vocal files, we also used convention like 1Drum based.wav, 1Monophony based.wav, 1Percusion based.wav, 1Polyphony based.wav, 1String 
based.wav, 1Woodwind based.wav, etc. IMAT provides three types of tempo tracing system that generates normal tempo or first multiplier or second multiplier or third multiplier of tempo of different instrumentals for the same vocal and search for an optimum combination between vocal and instrumental sounds. Select a vocal from a list of vocal performances and also select one tempo then play only vocal is depicted in the Figure 5. We select a particular vocal and then select a particular instrumental as well as a tempo and then play only instrumental is depicted in Figure 6. Finally we select a particular vocal and select multiple instrumentals and also select a particular tempo that is our final destination of optimum music composition is depicted in Figure 7. IMAT also provides a "save" button to save a particular music that is the combination of vocal and different types of instrumentals (ideal combination) in the Music Library.

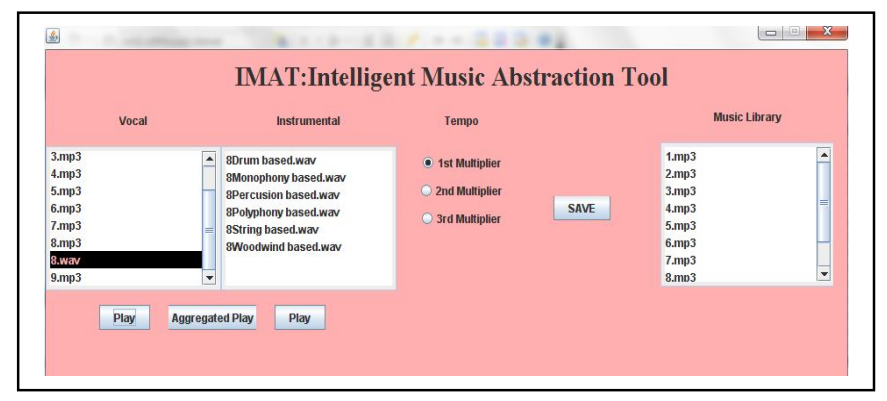

Figure 5: Snapshot of UID of IMAT that plays only Vocal

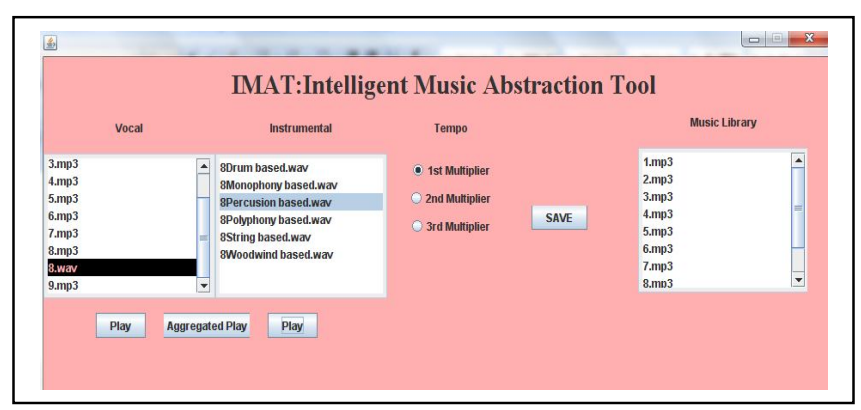

Figure 6: Snapshot of UID of IMAT that plays only Instrumental

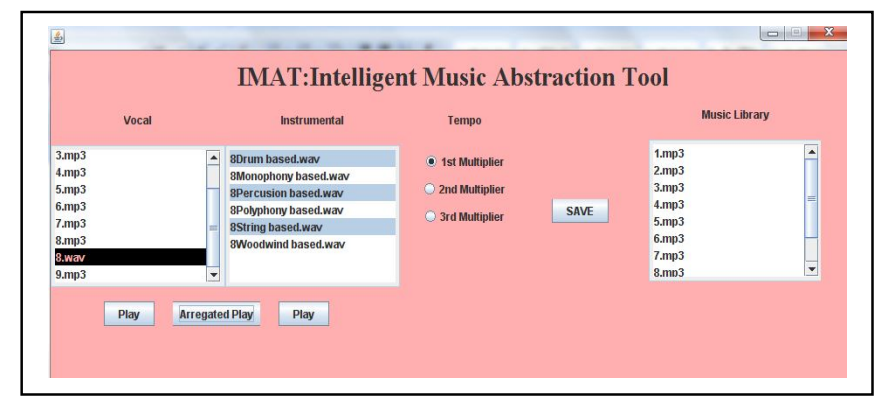

Figure 7: Snapshot of UID of IMAT that plays only Instrumental

\section{EXPERIMENTAL DETAILS}

The IMAT is a music software editor package that is used to provide music composition and performance improvisation system based on the concept of Software Engineering "Aggregation". In this article there are two music entities: Vocal and Instrumentals which will allow musicians to create an ideal combination of both and generate Music. This application is intended to enrich the possibilities of computer assisted music composition.

It has been conducted a comprehensive multiple state-of-the-art survey work to assess the utilization and effectiveness of IMAT. The primary aim of the survey work is to find out whether the IMAT is technically, operationally, and economically feasible or not. Twenty participants (10 men and 10 female, average aged between 20 to 40) took part in this analysis. Male participants and Female participants are represented by $\mathrm{M}$ and $\mathrm{F}$ respectively. Each participant gave written informed consent before taking part in this study. The experiment consisted of three parts: a training portion, a freeform composition portion, and a post-experiment paper questionnaire. Participants were introduced to the equipment and allowed to familiarize themselves with the music composition process using the object oriented concept of abstraction mechanism.

The overall experiment required approximately one hour. The freeform composition portion occurs after the training session has been completed. The questionnaire, using a $0-10$ Grading Scale, asked questions about the difficulty of the task, enjoyment of the task, Tempo Tracking of the composed music composition, features of music extension support files, and overall ratings by the each participants. The survey report given in the Table 2. Participant $M$ means Men and Participant F means Female.

Table 2: Survey Report of the user of IMAT (Here, $\mathrm{M}=$ Male Participants and $\mathrm{F}=$ Female Participants)

\begin{tabular}{|c|c|c|c|c|c|}
\hline Participants & Difficulty & Enjoyment & $\begin{array}{c}\text { Tempo } \\
\text { Tracking }\end{array}$ & $\begin{array}{c}\text { Music } \\
\text { File } \\
\text { Supports }\end{array}$ & Ratings \\
\hline M 1 & 1 & 7 & 7 & 9 & 8 \\
\hline M2 & 1.5 & 6.5 & 6 & 9 & 8 \\
\hline M3 & 0 & 7.8 & 8 & 10 & 8.5 \\
\hline M 4 & 1.2 & 9 & 8 & 10 & 9 \\
\hline M 5 & 1 & 10 & 8 & 10 & 9 \\
\hline M6 & 1 & 9 & 8 & 10 & 9 \\
\hline M7 & 2 & 8 & 7 & 9 & 8 \\
\hline M 8 & 0 & 8 & 9 & 10 & 8.5 \\
\hline M9 & 0 & 9 & 10 & 10 & 10 \\
\hline
\end{tabular}




\begin{tabular}{|c|c|c|c|c|c|}
\hline M 10 & 0 & 8 & 8.5 & 10 & 8.5 \\
\hline F1 & 1 & 7 & 8 & 9 & 8 \\
\hline F2 & 1.2 & 7.5 & 9 & 10 & 8.5 \\
\hline F3 & 1.5 & 8 & 7.5 & 9 & 8 \\
\hline F4 & 1.5 & 9 & 9 & 10 & 9 \\
\hline F5 & 1 & 9 & 10 & 10 & 10 \\
\hline F6 & 1 & 8 & 7 & 9 & 8 \\
\hline F7 & 2 & 8 & 7 & 10 & 8.5 \\
\hline F8 & 1 & 10 & 8 & 10 & 8.5 \\
\hline F9 & 1 & 6 & 8 & 9 & 8 \\
\hline F10 & 0 & 7 & 8 & 10 & 7.5 \\
\hline
\end{tabular}

Fig. 8 shows the distribution of Grading Scale by the each participant we received from the questionnaire session and it clearly shows that IMAT is a less difficult or complex, highly enjoyable, good variations of tempo tracking facilities, supports a great number of music files for generating music composition and overall rating of IMAT is remarkable by the participants. It provides a promising step toward creating an intelligent combination between vocal performances and instrumentals with different tempo for improvising the music composition based on the real time. Qualitative data from the above Fig. 8, it indicates that the IMAT is very simple that is not difficult interface for generating music improvisation, very good rating for both user enjoyment and applicable for tempo tracking, outstanding rating for both music file supports for IMAT and user' given rating. The combinations of these five grading features of IMAT, its user that is music composers used to create an intelligent innovative music editor that capable of creating unique melody which can constantly be updated or modified.

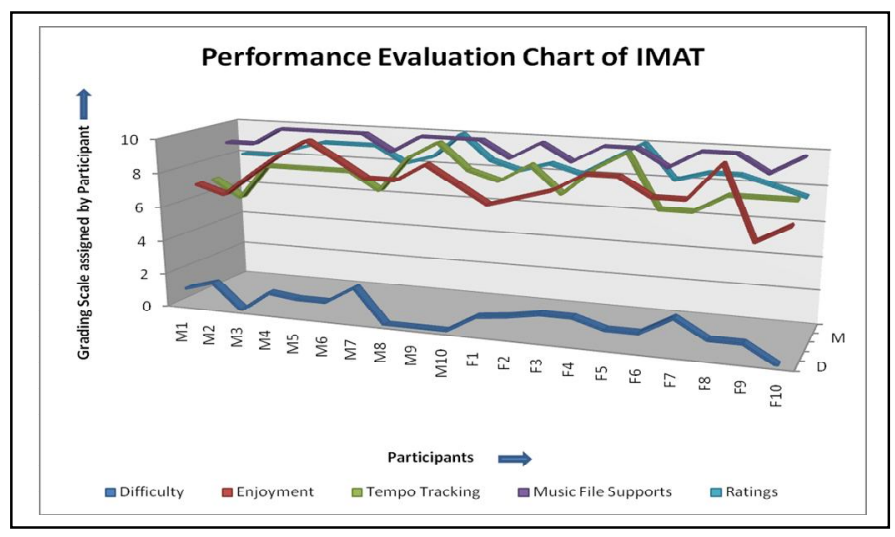

Figure 8: Performance Evaluation Chart of IMAT

\section{CONCLUSION}

Computer-assisted music composition is an emerging field. The traditional way of Music Composition is the application of computer to improvisation of innovative music composition through the use music software with some musical instruments like guitar, piano, ukulele, violin,

accordion, flute, keyboard, drum etc. The improvisation of Music Composition is a critical task that can be classified into major three categories - (1) Sound Oriented Composition, (2) Notation Oriented Composition, and (3) Analysis Oriented Composition. The work is fully based on sound oriented optimum combination. IMAT or Intelligent Music Abstraction Tool is a music editor that allows to the music composer to create different innovative music pieces by using some specific input vocal files and their corresponding different instrumental files and their one particular tempo. Innovative music composition process using the IMAT software solution by utilizing the optimum combination of vocal sound, tempo tracking, and instrumental sounds, becoming the one emerging tool for manipulating innovative music media editor.

The paper implemented as a step towards developing a music editor software tool to assist and evaluate the different musical pieces by combining the same vocal performance with different combinations of instrumentals pieces with different levels of tempo which is useful for different listeners for pleasant listening. The music editor software is very simple and user friendly as it supports both .mp3 files and .wav files for improvisation of music composition. In this paper, we do not incorporate the music features like score calculation, calculation of time signature, extraction of fundamental frequencies or note structures, etc. In future, we intended to combine all of the advanced types of musical features like polyphony score generation, generation of higher-level score structures for arranging of music elements, generation of relation and ordering musical gestures, real time music synthesis, extraction of music note structures using IMAT and evaluate each of the solutions again.

\section{ACKNOWLEDGEMENT}

We would like to express our sincere thank to both Techno Main Salt Lake and Sikkim Manipal Institute of Technology as we have used the infrastructures of these Institutes. We are also very much thankful for all our well wishers for their consistent support and encouragement.

\section{REFERENCES}

1. Clayton, M.: Time in Indian Music: Rhythm, Metre, and Form in North Indian Râg Performance, Oxford Monographs on Music. Oxford: Oxford University Press (2000).

2. Chordia, P., Rae, Alex: Tabla Gyan: A System for realtime tabla recognition and resynthesis, In Proceedings of the International Computer Music Conference, (2008).

3. Chordia, Parag: Automatic Transcription of Solo Tabla Music, Ph.D. diss., Stanford University, (2006).

4. Shmulevich, I., Yli-Harja, O., Coyle, E. J., Povel, D., Lemstrm, K.: Perceptual Issues in Music Pattern 
Recognition Complexity of Rhythm and Key Finding, Computers and the Humanities, Kluwer Academic Publishers, (2001).

5. Shmulevich, I., Povel, D.: Rhythm Complexity Measures for Music Pattern Recognition, In Proceedings of IEEE Workshop on Multimedia Signal Processing, (1998).

6. Gillet, O., Richard, G.: Automatic Labeling of Tabla Signals, In Proceedings of the International Conference on Music Information Retrieval (Baltimore, MD) (2003) 117-124.

7. Essid, S., Richard, G., David, B.: Musical instrument recognition based on class pair-wise feature selection. In Proceedings of International Conference on Music Information Retrieval, (2004).

8. De, Debashis, Roy, Samarjit: Polymorphism in Indian Classical Music: A Pattern Recognition Approach, In Proceedings of International Conference on Communications, Devices and Intelligent Systems (CODIS), IEEE, (2012) 612-615.

https://doi.org/10.1109/CODIS.2012.6422277

9. De, Debashis, Roy, Samarjit: Inheritance in Indian Classical Music: An Object-Oriented Analysis and Pattern Recognition Approach, In Proceedings of International Conference on Radar, Communication and Computing (ICRCC), IEEE, (2012) 193-198. https://doi.org/10.1109/ICRCC.2012.6450575

10. Chakraborty, Sayanti, De, Debashis: Object Oriented Classification and Pattern Recognition of Indian Classical Ragas, In Proceedings of the 1st International Conference on Recent Advances in Information Technology (RAIT), IEEE, (2012).

11. Chakraborty, Sayanti, De, Debashis: Pattern Classification of Indian Classical Ragas based on Object Oriented Concepts, In Proceedings of the International Journal of Advanced Computer engineering \& Architecture, Volume 2, (2012), 285-294.

12. Lande, T. S., Vollsnes, A. O.: Object Oriented Music Analysis, Computers and the Humanities 28 (1995) 253-257.

13. Bhattacharyya, M., De, D.: An Approach to identify Thhat of Indian Classical Music, In Proceedings of International Conference on Communications, Devices and Intelligent Systems (CODIS), IEEE, (2012) 592-595.

14. Datta, A. K., Sengupta, R., Dey, N., Nag, D.: Pitch Analysis of Recorded Vocal Performances in Hindustani Music: Evidence of a Personal Scale, J. Acoust. Soc. Ind., XXV, VI-1.1, (1997).

15. Datta, A. K., Sengupta, R., Dey, N., Nag, D., Mukherjee, A.: A Methodology of Note Extraction from the song signals, In Proceedings of the Yugoslav Journal of Operations Research, Volume 20, 1 (2010) 157-177. https://doi.org/10.2298/YJOR1001157M

16. Ellis, Dan: Pattern Recognition Applied to Music Signals, JHU CLSP Summer School, Laboratory for Recognition and Organization of Speech and Audio Columbia University, New York (2003).
17. Tzanetakis, George, Kapur, Ajay, Schloss, W. Andrew, Wright, Matthew: Computational Ethnomusicology, journal of interdisciplinary music studies, 1 (2007) 1-24,.

18. Schildt, Herbert: Java ${ }^{\mathrm{TM}}$ : The Complete Reference, Seventh Edition, Tata Mcgraw Hill Education Private Limited, New Delhi, India (2011).

19. Balagurusamy, E.: Programming with Java - A Primer, Fourth Edition, Tata Mcgraw Hill Education Private Limited, New Delhi, India (2009).

20. Chakrabarty, Sudipta, De, Debashis: Quality Measure Model of Music Rhythm using Genetic Algorithm, In Proceedings of International Conference on Radar, Communication and Computing (ICRCC), IEEE, (2012) 125-130.

21. Gartland-Jones, Andrew, Copley, Peter: The Suitability of Genetic Algorithms for Musical Composition, Contemporary Music Review, Vol. 22, 3 (2003) 43-55.

22. Matic, Dragan: A Genetic Algorithm for composing Music, In proceedings of the Yugoslav Journal of Operations Research, Volume 20, 1 (2010) 157-177. https://doi.org/10.2298/YJOR1001157M

23. Dostal, Martin: Genetic Algorithms as a model of musical creativity - on generating of a human-like rhythmic accompaniment, Computing and Informatics, 22 (2005) 321-340.

24. Chordia, Parag, Sastry, Avinash, Albin, Aaron: Evaluating Multiple Viewpoint Models of Tabla Sequences, In the Proceedings of 3rd International workshop on Machine learning and music, ACM (2010) 21-24.

25. Coyle, Edward J., Shmulevich, I.: A System for machine recognition of music patterns, In Proceedings of the Acoustic, Speech and Signal Processing, IEEE, 3 (1998) 3597-3600.

26. 26.Alfonceca, M., Cebrian, M., Ortega, A.: A Fitness Function for Computer-Generated Music using Genetic Algorithms, WSEAS Trans. On Information Science \& Applications, Issue 3, 3 (2006) 518-525.

27. Booch, G.,Object Oriented Analysis and Design with Applications. Addison-Wesley (1994).

28. Bruce, K van Gent, R., Schuett, A., PolyTOIL: A Type-Safe Polymorphic Object-Oriented language, in proceedings 9th European Conf. on O-O Programming (ECOOP'95), Springer, LNCS 952 (1995).

29. Claudia, Pons, Gabriel, Baum: Formal foundations of object-oriented modeling notations 3rd International Conference on Formal Engineering Methods, ICFEM 2000, York, UK.IEEE Computer Society Press. (2000).

30. Snoeck, Monique, Dedene, Guido: Generalization/Specialization and Role in Object-Oriented Conceptual Modeling,

31. Mall, Rajib: Fundamentals of Software Engineering, Third Edition, PHI Learning Pvt. Ltd., New Delhi, India (2010).

32. Claudia, Pons, Roxana, Giandini, Gabriel, Baum: Specifying Relationships between models through the software development process, Tenth International 
Workshop on Software specification and Design (IWSSD), San Diego, California, IEEE Computer Society Press. (2000).

33. UML, The Unified Modeling Language Specification Version 1.4, UML Specification, revised by the OMG, http://www.omg.org, September (2001).

34. Claudia, Pons, Generalization Relation in UML Model Elements, revised by the CiteSeerX, http://www.citeseerx.ist.psu.edu.

35. Szlenk, Marcin: Formal Semantics and Reasoning about UML Class Diagram, In Proceedings of the International Conference on Dependability of Computer Systems, DepCos-RELCOMEX-06, (2006) 51-59.

36. Samarjit Roy, Sudipta Chakrabarty, Pradipta Bhakta and Debashis De.: Modelling High Performing Music Computing using Petri Nets, Accepted In: International Conference on Control, Instrumentation, Energy and Communication (CIEC), IEEE, pp. 757-761, (2013).

37. Samarjit Roy, Sudipta Chakrabarty and Debashis De.: A Framework of Musical Pattern Recognition using Petri Nets, Accepted In: Emerging Trends in Computing and Communication (ETCC), Springer-Link Digital Library, Submission No.- 60, (2013).

38. Sudipta Chakrabarty, Samarjit Roy and Debashis De.: Pervasive Diary in Music Rhythm Education: A Context-Aware Learning Tool using Genetic Algorithm, Accepted in: Springer- Verlag, Smart Innovation, Systems and Technologies, ISSN: 2190-3018, Submission No.- ICACNI-271, (2014). https://doi.org/10.1007/978-3-319-07353-8_77

39. Sudipta Chakrabarty, Samarjit Roy and Debashis De.: Pervasive Diary in Music Rhythm Education: A Context-Aware Learning Tool using Genetic Algorithm, Accepted in: Springer- Verlag, Smart Innovation, Systems and Technologies, ISSN: 2190-3018, Submission No.- ICACNI-271, (2014).

40. Seungmin Rho, Byeong-jun Han, Eenjun Hwang, Minkoo Kim. :MUSEMBLE: A novel music retrieval system with automatic voice query transcription and reformulation, Journal of Systems and Software, Elsevier, pp. 1065-1080, (2008).

41. Chakrabarty Sudipta, Islam Md Rahul, Debasarma Hiren Kumar, "An approach towards the modeling of Pattern Similarity in Music using Statistical Measures" Accepted ehe paper in the 5th International Conference on Parallel, Distributed and Grid Computing (PDGC), in JUIT, Waknaghat, Himachal Pradesh, 2018.

42. Chakrabarty Sudipta, Banik Sangeeta, Islam Md Rahul, Debasarma Hiren Kumar, "Context Aware Song Recommendation System", accepted the paper in the 3rd National Conference on Communication, Cloud, and Big Data (CCB), in SMIT, Majhitar, East Sikkim, 2018.

43. Chakrabarty Sudipta, Islam Md Ruhul, De Debashis, "Modelling of Song Pattern Similarity using Coefficient of Variance", International Journal of Computer Science and Information Sequrity (ISSN 1947-5500), pp. 388-394, 2017.
44. Roy Samarjit, Chakrabarty Sudipta, De Debashis, "Time-Based Raga Recommendation and Information Retrieval of Musical Patterns in Indian Classical Music using Neural Network", IAES International Journal of Artificial Intelligence (IJ-AI) (ISSN: 2252-8938), pp. 33-48, 2017.

https://doi.org/10.11591/ijai.v6.i1.pp33-48

45. Chakrabarty Sudipta, Roy Samarjit, De Debashis, Edited by Siddhartha Bhattacharyya, Hrishikesh Bhaumik, Sourav De, Goran Klepac, Handbook of Research on Intelligent Analysis of Multimedia information (Hardcover), Chapter 12: Time-Slot Based Intelligent Music Recommender in Indian Music, ISBN13: 9781522504986, ISBN10: 1522504982, Publisher: IGI Global, USA, pp. , 2016.

46. Chakrabarty Sudipta, Karmakar Gobinda, Islam Md Rahul, De Debashis, "Raconing of Music Rhythm Density and Complexity through Mathematical Measures", In Proceedings of the Advanced Computational and Communication Paradigm, LNEE Lecture Note, Springer, pp. , 2017.

47. Chakrabarty Sudipta, Roy Samarjit, De Debashis, "A Foremost Survey on State-Of-The-Art Computational Music Research", In Proceedings of the Recent Trends in Computations and Mathematical Analysis in Engineering and Sciences", International Science Congress Association, pp. 16-25, 2015.

48. Chakrabarty Sudipta, Samarjit Roy, debashis De, "Behavioural Modelling of Ragas of indian Classical Music using Unified Modelling Language", In Proceedings of the 2nd International Conference on Perception and Machine Intelligence, ACM, pp. 151-160, 2015. https://doi.org/10.1145/2708463.2709037

49. Sudipta Chakrabarty, Roy Samarjit, De Debashis, "Automatic Raga Recognition using Fundamental Frequency Range of Extracted Musical Notes", In the Proceedings of Eight International MultiConference on Image and Signal Processing (ICISP 2014), Elsevier, pp. 337-345, 2014.

50. Mukkamala S N V Jitendra, Dr Y Radhika, "A Review: Music Feature Extraction from an Audio Signal", International Journal of Advanced Trends in Computer Science and Engineering, Vol. 9, No. 2, Warse, pp. 973-980, 2020. https://doi.org/10.30534/ijatcse/2020/11922020

51. Sandip Dalal, Kamna Solanki, Sudhir, Diksha, "Exploring the Essentials and Principles of Software Development", International Journal of Advanced Trends in Computer Science and Engineering, Vol. 8, No. 6, Warse, pp. 3504-3510, 2019. https://doi.org/10.30534/ijatcse/2019/129862019 